\title{
Estudo da Intensidade de Campo Elétrico na Região Geométrica Central de Micro/Nano-antenas Tipo Dipolo em $29,9 \mathrm{THz}$
}

\author{
Liangrid Lutiani Silva ${ }^{1}$ \\ Instituto Tecnológico da Aeronáutica, ITA, São José dos Campos, SP \\ Newton Adriano dos Santos Gomes ${ }^{2}$ \\ Instituto Tecnológico da Aeronáutica, ITA, São José dos Campos, SP \\ Vilson Rosa Almeida ${ }^{3}$ \\ Instituto Tecnológico da Aeronáutica, ITA, São José dos Campos, SP \\ Instituto de Estudos Avançados, IEAv, São José dos Campos, SP
}

\begin{abstract}
Resumo. O presente trabalho é um estudo prospectivo relacionado ao entendimento dos efeitos de variações de geometria em antenas tipo dipolo, projetadas para operar na faixa de frequência Terahertz ( $\mathrm{THz}$ ), nos valores de amplitude de campo elétrico na região central das antenas $(G A P)$. A identificação e a correta otimização da geometria das antenas devem ser cuidadosamente consideradas de modo a maximizar o campo elétrico na região de interface entre a antena e o diodo retificador MIM, o que pode vir a prover melhores características de geração de corrente elétrica, em especial, nos efeitos de corrente por mecanismos de tunelamento.
\end{abstract}

Palavras-chave. micro/nano-antenas, detecção THz, coleta de energia, corrente de tunelamento, fotônica em silício, diodos MIM.

\section{Introdução}

O potencial emprego de micro/nano-antenas operando na faixas de frequências Terahertz $(\mathrm{THz})$, bem como ópticas, tem recebido atenção especial de grupos de pesquisa ao redor do globo, principalmente no que tange a aplicações de detecção de ondas $\mathrm{THz}[1]$, detecção de fótons [2], coleta de energia (energy harvesting) [3], sistemas de comunicações wireless [4], bem como no emprego em sistemas de comunicação intra-chip [5], [6], os quais visam compor avançadas malhas de comunicação de dados, em circuitos integrados que manipulam informações empacotadas tanto no domínio fotônico quanto no elétrico, concomitantemente.

\footnotetext{
${ }^{1}$ liangrid@ita.br

${ }^{2}$ newtonsg@ita.br

${ }^{3}$ vilsonra@ita.br
} 
As denominadas rectennas (micro/nano-antenas dispostas conjuntamente com um diodo retificador) compõem um sistema de conversão de energia, onde uma onda eletromagnética propagando-se no espaço livre é transformada em um sinal elétrico com forma de onda alterada (A.C.) que, por sua vez, é convertido (retificado) por meio de um diodo (geralmente um diodo tipo Metal-Isolante-Metal (MIM)), em um sinal elétrico continuo (D.C.). Diversas configurações desse sistema vêm sendo estudados, principalmente para aplicações de detecção e captura de energia $[7,8]$. Um dos principais pontos a serem considerados no design de rectennas está relacionado com a interface antena-diodo, onde entre outras características, a intensidade de campo elétrico nessas interfaces apresenta um papel importante na estimativa da corrente elétrica que efetivamente será retificada [9]. O presente estudo visa verificar a influência dos efeitos da geometria de uma antena dipolo projetada para operar em 29,9 THz $(\lambda=10 \mu \mathrm{m})$, constituída de materiais distintos em cada uma das hastes da antena dipolo: alumínio $(\mathrm{Al})$ e cobre $(\mathrm{Cu})$. O prospectivo estudo demostra, por meio de simulação, via FEM (acrônimo do idioma inglês para: Finite Element Method), a dependência da amplitude do campo elétrico em relação às espessuras e ao formato das hastes das antenas, uma vez que os diodos MIM operam nesse regime de frequências basicamente por mecanismos de corrente de tunelamento [10,11]. Cabe ainda salientar que o diodo MIM não está contemplado no presente estudo, mas a influência do campo elétrico é um importante condicionante na quantificação das características de operação e projeto dos mesmos [10]. Contudo, existem outras variáveis de influência, como casamento de impedâncias, área da seção do diodo, dentre outros $[9,10]$, que não são abordadas neste trabalho. A seguir, são apresentados os materiais e métodos utilizados no estudo e, em seguida, são apresentadas as seções de resultados e conclusões.

\section{Materiais e Métodos}

Visamos avaliar a dependência da amplitude do campo elétrico entre as interfaces das hastes de duas antenas com geometrias ligeiramente distintas, por meio da variação paramétrica da espessura de uma das hastes de cada uma das antenas $\left(L_{m 1}\right)$ (vide tabela 1). As duas geometrias de antenas dipolo analisadas diferem uma da outra pela terminação das hastes, onde uma delas termina geometricamente em uma seção plana, Figura 1-(a), enquanto que a outra tem sua terminação com raios de curvatura, como representado na Figura 1-(b). A Figura 1-(c) apresenta uma visão da seção transversal dos modelos. As antenas foram modeladas considerando sua disposição sob uma camada de Óxido de Silício $\left(\mathrm{SiO}_{2}\right)$, com metalização na superfície superior. A estrutura de suporte foi definida como Silício ( $\mathrm{Si}$ ). Os principais parâmetros geométricos dos modelos simulados são apresentados na tabela 1. Cabe destacar que as dimensões laterais da estrutura de suporte (camadas de $\mathrm{SiO}_{2}$, metalização e $\mathrm{Si}$ ) são iguais, i.e. quadrada de comprimento $L S$. O espaço entre os dipolos foi denominado de $(G A P)$. Ainda, a região do GAP é assumida como a região de interface entre a antena e o diodo MIM.

Definiu-se que as antenas são exitadas por uma onda plana incidente, propagando-se na direção $-z$ com polarização linear na direção $x$ (orientação do sistema de coordenadas, vide Figura 1), e amplitude de campo elétrico de $100 \mathrm{mV} / \mathrm{m}$. 


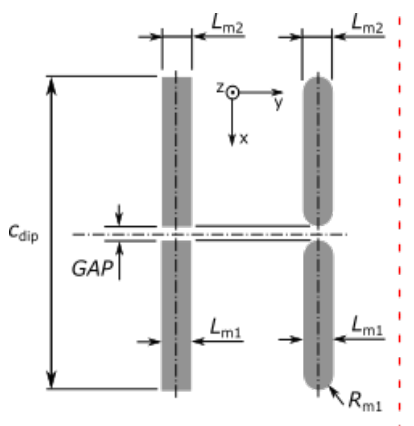

(a)

(b)

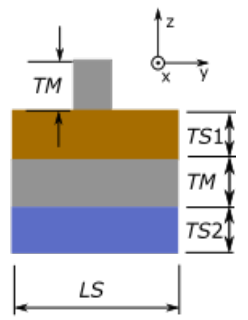

(c)

Figura 1: Ilustração das estruturas em estudo. (a) antena dipolo com terminação por seções planas; (b) antena dipolo com terminações em raio; (c) vista lateral da estrutura.

Exceto quanto ao material no entorno das antenas, definido como sendo preenchidas por ar atmosférico (ar), as demais propriedades elétricas e ópticas como permissividade elétrica relativa, condutividade elétrica e tangente de perdas aplicadas aos modelos, são dependentes da frequência e; assume-se estar sob temperatura ambiente em $(T=293 \mathrm{~K})$. Nos metais, $\mathrm{Al}$ e $\mathrm{Cu}$, foram definidos de acordo com o trabalho de Rakić [12] e Johnson e Christry [13], respectivamente. As camadas de $\mathrm{SiO}_{2}$ e $\mathrm{Si}$ foram assumidas de acordo com o trabalho de Palik [14].

As simulações computacionais foram realizados com software HFSS ${ }^{\circledR}$, um software baseado em elementos finitos (Finite Element Method - FEM) especializado em solucionar, de forma completa, os campos eletromagnéticos, no espaço tridimensional, dados pelas equações de Maxwell. As simulações foram resolvidas por meio de malha adaptativa, com refino de malha na região do GAP de cada antena. O critério de convergência foi definido em termos de nível de energia e o critério de parada definido em 15 passos de cálculo.

Tabela 1: Categorias dos trabalhos.

\begin{tabular}{|c|c|c|}
\hline Parâmetro & Dimensão da Antena (a) & Dimensão da Antena (b) \\
\hline$L_{m 2}$ & $300 \mathrm{~nm}$ & $300 \mathrm{~nm}$ \\
\hline$L_{m 1}$ & $\in\{300,250,200,150,100\} n \mathrm{~nm}$ & $\in\{300,250,200,150,100\} \mathrm{nm}$ \\
\hline$R_{m 1}$ & - & $\mathrm{L}_{m 1} / 2$ \\
\hline$c_{d i p}$ & $2.5 \mu \mathrm{m}$ & $2.5 \mu \mathrm{m}$ \\
\hline$G A P$ & $5 \mathrm{~nm}$ & $5 \mathrm{~nm}$ \\
\hline$T M$ & $300 \mathrm{~nm}$ & $300 \mathrm{~nm}$ \\
\hline$T S 1$ & $2.3 \mu \mathrm{m}$ & $2.3 \mu \mathrm{m}$ \\
\hline$T S 2$ & $380 \mu \mathrm{m}$ & $380 \mu \mathrm{m}$ \\
\hline$L S$ & $10 \mu \mathrm{m}$ & $10 \mu \mathrm{m}$ \\
\hline
\end{tabular}

Em ambos os casos estudados, foi definido por conveniência que o material da haste que varia de espessura é o Al. A seguir são apresentados os resultados do estudo. 


\section{Resultados}

A intensidade dos campos foi avaliada assumindo-se a direção $x$ do $G A P$, posicionada exatamente na linha de simetria central das hastes das antenas. Na Figura 2 é evidenciada a dependência da intensidade do campo elétrico em relação à largura da seção lateral da haste da antena (Figura 1-(a)). A medida em que $L_{M 1}$ é reduzido a intensidade do campo elétrico é ampliada.

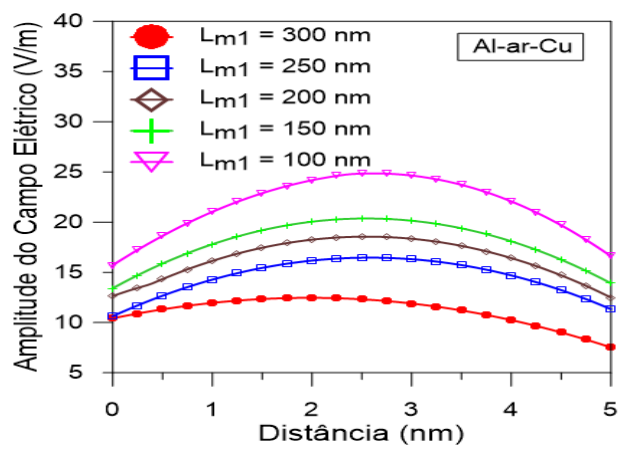

Figura 2: Intensidade de campo elétrico em função do comprimento do GAP, para as diferentes espessuras da haste da antena com terminação em seção reta.

Comportamento análogo é verificado na Figura 2, representando a variação de $L_{M 1}$ na antena com terminações em forma de raio (Figura 1-(b)). Contudo, os valores de intensidade da campo elétrico nas regiões com raio de curvatura são superiores aos demonstrados no caso de hastes com terminação reta. Esse fato se deve ao aumento do acúmulo de cargas devido a uma crescente elevação da divergência do campo elétrico, em conformidade com a equação de Gauss.

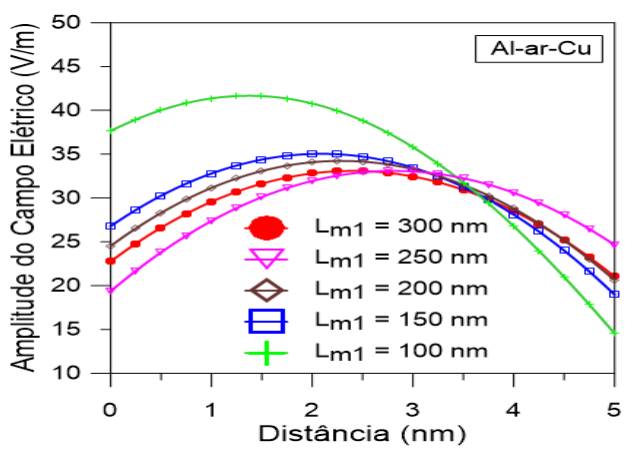

Figura 3: Intensidade de campo elétrico em função do comprimento do GAP, para as diferentes espessuras da haste da antena com terminação em geométrica em raio. 


\section{Conclusões}

Por meio de simulação computacional foi verificado que a intensidade do campo elétrico na região do GAP das antenas estudadas é dependente da espessura das hastes, bem como da geometria das bordas. Essa verificação e otimização são importantes para maximizar a transferência de cargas por mecanismos de tunelamento em diodos MIM [10]. De fato, em situações práticas, a geometria apresentada na figura 1-(a) é de difícil obtenção em escalas nanométricas, mesmo por meio da aplicação dos melhores processos de microfabricação existentes. Isso decorre de defeitos inerentes aos mesmos, como resolução de máscaras de fabricação e não podem ser totalmente excluídos, mas podem ser mitigados [11]. Pretendese, em trabalhos em andamento, abordar outros aspectos relevantes, incluindo simulações em uma faixa de frequências em torno da frequência central de operação das antenas, de modo a aprofundar o entendimento das características de interface entre antena e diodo, bem como do mecanismo de corrente de tunelamento em diodos MIM, acoplados às antenas.

\section{Agradecimentos}

Este trabalho é suportado pela CAPES. L.L.S. agradece a CAPES pela bolsa de estudos e V.R.A. agradece ao CNPq, processo $n^{\circ}$ 310855/2016-0 e a CAPES: bolsa PVS CAPES/ITA, Edital no 48/2014. Os autores agradecem ao lab. LEC/IEAv pelo recurso computacional disponibilizado para realização deste trabalho.

\section{Referências}

[1] M. N. Gadalla and A. Shamim. 28.3 THz Bowtie Antenna Integrated Rectifier for Infrared Energy Harvesting. In Proceedings of the 44th European Microwave Conference, p. 652-655, 2014.

[2] F. Bigourdan, F. Marquier, J.-P. Hugonin, and J.-J. Greffet. Design of highly efficient metallo-dielectric patch antennas for single-photon emission. Opt. Express, vol. 22, no. 3, p. 2337-47, 2014.

[3] Y. M. El-Toukhy, M. Hussein, M. F. O. Hameed, A. M. Heikal, M. M. Abd-Elrazzak, and S. S. A. Obayya. Optimized tapered dipole nanoantenna as efficient energy harvester. Opt. Express, vol. 24, no. 14, p. A1107, 2016.

[4] K. Takahashi, T. Higashino, T. Nakamura, Y. Aburakawa, K. Tsukamoto, S. Komaki, K. Wakamori, T. Suzuki, K. Kazaura, A. M. Shah, K. Omae, M. Matsumoto, and Y. Miyamoto. Design and evaluation of optical antenna module suitable for radio-on free-space optics link system for ubiquitous wireless. Proceeding SPIE, vol. 6877, no. 2008, p. 1-11, 2008. 
[5] H. Zhou, S. Member, Z. Li, S. Member, L. Shang, A. Mickelson, S. Member, D. S. Filipovic, and S. Member. On-Chip Wireless Optical Broadcast. J. Light. Technol., vol. 28, no. 24, p. 3569-3577, 2010.

[6] H. Zhou, X. Chen, D. S. Espinoza, A. Mickelson, and D. S. Filipovic. Nanoscale optical dielectric rod antenna for on-chip interconnecting networks. IEEE Trans. Microw. Theory Tech., vol. 59, no. 10, p. 2624-2632, 2011.

[7] M. N. Gadalla, M. Abdel-Rahman, and A. Shamim. Design, optimization and fabrication of a $28.3 \mathrm{THz}$ nano-rectenna for infrared detection and rectification. Sci. Rep. , vol. 4, p. 4270, 2014.

[8] E. Briones, J. Alda, and F. J. González. Conversion efficiency of broad-band rectennas for solar energy harvesting applications.Opt. Express, vol. 21, p. A412-8, 2013.

[9] S. Grover and G. Moddel. Engineering the current-voltage characteristics of metalinsulator-metal diodes using double-insulator tunnel barriers. Solid. State. Electron., vol. 67, no. 1, p. 94-99, 2012.

[10] J. G. Simmons. Conduction in thin dielectric films. J. Phys. D. Appl. Phys., vol. 4, no. 5 , p. 630, 1971.

[11] K. Choi, F. Yesilkoy, G. Ryu, S. H. Cho, N. Goldsman, M. Dagenais, and M. Peckerar. A focused asymmetric metal-insulator-metal tunneling diode: Fabrication, DC characteristics and RF rectification analysis. IEEE Trans. Electron Devices, vol. 58, no. 10, p. 3519-3528, 2011.

[12] A. D. Rakić. Algorithm for the determination of intrinsic optical constants of metal films: application to aluminum. Appl. Opt., vol. 34, no. 22, p. 4755, 1995.

[13] P. B. Johnson and R. W. Christry. Optical Constants of the Noble Metals. Phys. Rev. $B$, vol. 6 , no. 12 , p. 4370-4379, 1972 .

[14] E. D. Palik. Handbook of Optical Constants of Solids. Academic Press. New York, 1985. 